\title{
Préparer le terrain pour l'élargissement de I'utilisation de la prophylaxie préexposition contre le VIH au Canada
}

\author{
M Hull ${ }^{1,2, *}$, DHS Tan ${ }^{3}$
}

\section{Résumé}

Le virus de l'immunodéficience humaine $(\mathrm{VIH})$ continue d'affecter de façon disproportionnée les populations vulnérables au Canada, particulièrement les hommes qui ont des rapports sexuels avec des hommes (HARSAH). Les stratégies de prévention du VIH ont récemment élargi leur champ d'application : elles sont passées de la simple prophylaxie post-exposition non professionnelle (nPEP) à la suite d'expositions à haut risque à la prophylaxie préexposition (PrEP) grâce à laquelle on réduit son risque d'infection au VIH en utilisant une combinaison d'antirétroviraux, pris avant l'exposition au risque. Compte tenu du fait qu'on n'a approuvé I'usage du ténofovir et de l'emtricitabine (TDF/FTC) pour la PrEP qu'au début de 2016 et que le financement public est encore limité, l'adoption au Canada est à un stade préliminaire. Ces stratégies de prévention biomédicale ont prouvé l'efficacité pour les HARSAH et elles peuvent présenter un potentiel pour d'autres populations à risque. Avec les formulations génériques de TDF/FTC maintenant offertes au Canada, il est possible d'effectuer une vaste mise en œuvre. L'élargissement des connaissances des fournisseurs de soins de santé dans tout le Canada sur la meilleure façon d'évaluer, de prescrire, de recommander et de surveiller la PrEP contribuera aux efforts déployés actuellement pour atteindre l'objectif mondial d'éliminer les nouvelles infections au $\mathrm{VIH}$.

\author{
Affiliations \\ ${ }^{1}$ BC Centre for Excellence \\ in HIV/AIDS, Vancouver \\ (Colombie-Britannique) \\ 2 Université de la \\ Colombie-Britannique, Vancouver \\ (Colombie-Britannique) \\ ${ }^{3}$ Faculté de médecine, Université \\ de Toronto, Toronto (Ontario)
}

*Correspondance: mhull@cfenet. ubc.ca

Citation proposée : Hull M, Tan DHS. Préparer le terrain pour l'élargissement de l'utilisation de la prophylaxie préexposition contre le VIH au Canada. Relevé des maladies transmissibles au Canada. 2017;43(12):310-7. https://doi.org/10.14745/ccdr.v43i12a05f

\section{Introduction}

II y a plus de 40 ans que l'épidémie de VIH a tout d'abord attiré l'attention des cliniciens qui avaient découvert des cas d'infections et de cancers opportunistes touchant des hommes jeunes et en bonne santé, qui étaient gays ou bisexuels ou qui avaient des rapports sexuels avec d'autres hommes (HARSAH) $(1,2)$. En dépit d'énormes progrès dans le diagnostic du VIH, des soins et de la gestion du virus, et de trois décennies de campagnes éducatives, les HARSAH continuent à porter le fardeau de l'épidémie de VIH au Canada (3). Les HARSAH au Canada sont maintenant 171 fois plus susceptibles de contracter I'infection que les hommes de la population générale. La plupart de ces infections sont maintenant concentrées dans les zones urbaines où, dans des villes comme Vancouver et Toronto, les HARSAH représentent toujours environ $70 \%$ de tous les nouveaux diagnostics de VIH chaque année (4). En outre, le Canada a connu une hausse spectaculaire des nouveaux diagnostics de VIH chez les Autochtones des Prairies, où ils sont associés à l'utilisation de drogues injectables (UDI) et à la transmission hétérosexuelle (5).

Dans certains pays, comme l'Angleterre qui a commencé à déployer des stratégies de prévention biomédicale telles que la prophylaxie préexposition (PrEP), une baisse importante du nombre de nouvelles infections a été constatée, surtout chez les HARSAH (6). La preuve de l'efficacité et les résultats prometteurs de la prise précoce à la fois de la prophylaxie préexposition et prophylaxie post-exposition pour prévenir la transmission du VIH constituent une nouvelle occasion importante pour les stratégies de prévention du VIH au Canada.

\section{Contexte}

Les stratégies de prévention du VIH ont traditionnellement misé sur les interventions comportementales individuelles, telles que l'amélioration de l'utilisation de préservatifs ou le counseling en santé sexuelle, ainsi que sur des interventions sur le plan communautaire afin de réduire le risque pour les populations marginalisées, comme l'échange de seringues ou des services de réduction des risques pour les personnes qui s'injectent des drogues (7). L'émergence de l'amélioration des stratégies de dépistage du VIH, y compris des essais biologiques comportant des fenêtres sérologiques plus courtes ou des points de prestation de soins de santé, a permis de diagnostiquer les infections au VIH plus tôt au début de la maladie, avec le potentiel de limiter la transmission ultérieure du virus. La thérapie antirétrovirale suppressive (TA) s'est avérée être efficace pour empêcher la transmission du VIH d'une personne séropositive à un partenaire sexuel non infecté $(8,9)$. L'expansion des programmes de TA, en combinaison avec des programmes de réduction des risques, a considérablement réduit les infections au $\mathrm{VIH}$ chez les consommateurs de drogues injectables 
dans le centre-ville de Vancouver, où l'on a observé une chute de nouveaux diagnostics, qui sont passés de plus de 350 en 1996 à moins de 30 en 2012 (10).

Malgré l'expansion considérable de la TA au Canada, la transmission sexuelle avant le diagnostic, en particulier chez les HARSAH affectés d'une séroconversion aiguë, contribue à l'épidémie sévissant au Canada (11-14). Il existe un consensus mondial émergent indiquant qu'une approche combinée regroupant les stratégies de prévention actuelles avec les interventions de prévention biomédicales émergentes offre le meilleur espoir de réduire les nouvelles infections au VIH (15). L'objectif de cet article est de décrire les traitements à la PrEP et à la prophylaxie post-exposition non professionnelle (nPEP), ainsi que les défis et occasions pour ces stratégies biomédicales de prévention du VIH qui ont le potentiel de modifier la trajectoire de l'épidémie du VIH au Canada et dans le monde.

\section{Stratégies de traitement à la prophylaxie préexposition et à la prophylaxie post-exposition}

Le recours à des médicaments antirétroviraux pour la prophylaxie post-exposition est, depuis des décennies, la norme en cas d'exposition à risque élevé se produisant dans un contexte professionnel (comme dans les hôpitaux). Leur utilisation pour les expositions à risque élevé, telles que l'exposition sexuelle consensuelle ou le partage de seringues (nPEP), est approuvée par des lignes directrices aux États-Unis depuis 2005 (16) et devenue la norme des soins dans la plupart des pays développés. Les personnes ayant un potentiel d'exposition au risque sont évaluées en fonction de la probabilité que la source de l'exposition soit séropositive et le risque de la nature de l'exposition elle-même. Si la source n'est pas considérée comme séropositive, la prévalence de l'épidémiologie locale du $\mathrm{VIH}$ parmi les populations à risque, telles que les HARSAH et les personnes s'injectant des drogues, est importante pour évaluer le risque. Les expositions à risque élevé incluraient le partage de seringues, les rapports sexuels anaux réceptifs sans protection; l'exposition à risque modéré comprend les rapports anaux ou vaginaux pénétrants sans protection ou les rapports vaginaux réceptifs sans protection. Les personnes ayant été exposées au risque modéré ou élevé d'une source ayant une forte probabilité de séropositivité sont admissibles à la nPEP. Dans ce cadre, les particuliers reçoivent une combinaison standard de médicaments antirétroviraux pendant un cycle de 28 jours afin de diminuer la probabilité d'infection. La prise des médicaments doit commencer dès que possible après l'exposition et au plus tard 72 heures après l'exposition (17). Ils doivent se soumettre à un dépistage du VIH 12 semaines après l'exposition pour s'assurer qu'ils sont séronégatifs et devraient se faire immédiatement recommander un traitement du VIH s'ils sont séropositifs.

En revanche, la PrEP fait référence à I'utilisation quotidienne d'une combinaison à dose fixe de fumarate de ténofovir disoproxil (TDF) et d'emtricitabine (FTC) conjointement avec des pratiques sexuelles plus sûres chez les personnes séronégatives. Ici, le traitement est utilisé sur une base continue, avant une éventuelle exposition au VIH, et poursuivie pour prévenir I'infection. Les personnes à risque potentiel d'infection devraient subir une évaluation de base pour s'assurer qu'elles sont séronégatives. L'utilisation d'une quatrième génération d'essai biologique du VIH est recommandée pour le dépistage en raison de sa fenêtre sérologique réduite. Les personnes montrant des signes ou des symptômes d'une infection au VIH, ou exposées à l'intérieur de la fenêtre sérologique (jusqu'à 21 jours après l'exposition) de l'essai biologique, doivent subir un test d'acide ribonucléique, un test d'amplification des acides nucléiques ou d'autres tests, ou encore être soumises à un test de vérification de 14 à 21 jours plus tard pour confirmer les résultats. Les personnes atteintes d'une infection au VIH non diagnostiquée qui commencent à prendre la PrEP risquent d'acquérir une résistance aux médicaments du $\mathrm{VIH}$, puisqu'elles ne reçoivent pas de traitement antirétroviral triple standard. II faut leur faire comprendre l'importance de s'y conformer rigoureusement pour bénéficier d'une protection complète et que les niveaux de protection sont atteints après sept jours d'utilisation quotidienne. La PrEP est très bien tolérée, avec un faible risque d'effets secondaires gastro-intestinaux dans les premières semaines et un risque potentiel de toxicité rénale et osseuse réversible. Une méta-analyse de 10 essais contrôlés par placebo a montré que la fréquence des événements indésirables est similaire au placebo $(R C=1,01, I C$ à $95 \%: 0,99$ à 1,03) (18). Les personnes ayant recours à la PrEP font l'objet d'un suivi régulier tous les trois mois, et l'on continue de dépister le VIH chez elles et l'on procède à des tests de leur fonction rénale et d'éventuelles infections transmissibles sexuellement. Voir le tableau 1 pour un résumé des traitements contre le $\mathrm{VIH}$ à la prophylaxie préexposition et à la prophylaxie post-exposition.

Tableau 1 : Sommaire des traitements contre le VIH à la prophylaxie préexposition et à la prophylaxie post-exposition

\begin{tabular}{|c|c|c|}
\hline Description & $\begin{array}{l}\text { Prophylaxie } \\
\text { préexposition } \\
\text { (PrEP) }\end{array}$ & $\begin{array}{l}\text { Prophylaxie post- } \\
\text { exposition non } \\
\text { professionnelle (nPEP) }\end{array}$ \\
\hline $\begin{array}{l}\text { Médicaments } \\
\text { approuvé }\end{array}$ & Oui & Non \\
\hline Population & $\begin{array}{l}\text { Personne à } \\
\text { haut risque } \\
\text { d'exposition } \\
\text { au VIH par des } \\
\text { rapports sexuels } \\
\text { ou le partage de } \\
\text { seringues }\end{array}$ & $\begin{array}{l}\text { IPersonne qui a été exposée, } \\
\text { au cours des } 72 \text { heures } \\
\text { précédentes, à un risque élevé } \\
\text { ou modéré par une personne } \\
\text { représentant un risque } \\
\text { important d'être victime d'une } \\
\text { infection au VIH transmissible }\end{array}$ \\
\hline $\begin{array}{l}\text { Médicaments } \\
\text { utilisés }\end{array}$ & $\begin{array}{l}\text { Fumarate } \\
\text { de ténofovir } \\
\text { disoproxil } \\
\text { et emtricitabine }\end{array}$ & $\begin{array}{l}\text { Des données variables, mais } \\
\text { à jour, viennent appuyer la } \\
\text { combinaison 1) du fumarate } \\
\text { de ténofovir disoproxil, 2) } \\
\text { de l'emtricitabine ou de la } \\
\text { lamivudine et 3) d'un inhibiteur } \\
\text { de l'intégrase (raltégravir ou } \\
\text { dolutégravir) ou d'un inhibiteur } \\
\text { de la protéase rehaussé } \\
\text { (darunavir ou ritonavir) }\end{array}$ \\
\hline Durée & $\begin{array}{l}\text { Indéfinie pendant } \\
\text { que le risque } \\
\text { d'exposition se } \\
\text { poursuit } \\
\text { Les médicaments } \\
\text { sont pris tous les } \\
\text { jours }\end{array}$ & Tous les jours pendant 28 jours \\
\hline
\end{tabular}


Tableau 1 : Sommaire des traitements contre le VIH à la prophylaxie préexposition et à la prophylaxie post-exposition (suite)

\begin{tabular}{|l|l|l|}
\hline Description & $\begin{array}{c}\text { Prophylaxie } \\
\text { préexposition } \\
\text { (PrEP) }\end{array}$ & \multicolumn{1}{|c|}{$\begin{array}{c}\text { Prophylaxie post- } \\
\text { exposition non } \\
\text { professionnelle (nPEP) }\end{array}$} \\
\hline $\begin{array}{l}\text { Effets } \\
\text { indésirables } \\
\text { courants }\end{array}$ & $\begin{array}{l}\text { Nausées, troubles } \\
\text { gastro-intestinaux, } \\
\text { faible risque de } \\
\text { néphrotoxicité } \\
\text { réversible et } \\
\text { diminution de la } \\
\text { densité osseuse }\end{array}$ & $\begin{array}{l}\text { Régime spécifique, mais peut } \\
\text { inclure des nausées, des maux } \\
\text { de tête, des troubles gastro- } \\
\text { intestinaux, un risque rare de } \\
\text { toxicité rénale ou hépatique, } \\
\text { un risque d'interactions des } \\
\text { médicaments si des inhibiteurs } \\
\text { de la protéase rehaussés sont } \\
\text { utilisés }\end{array}$ \\
\hline Surveillance & $\begin{array}{l}\text { Dépistage de base } \\
\text { du VIH et des ITS, } \\
\text { et bilans hépatique } \\
\text { et rénal } \\
\text { Tests de suivi } \\
\text { un mois après } \\
\text { l'utilisation et tous } \\
\text { les trimestres par } \\
\text { la suite }\end{array}$ & $\begin{array}{l}\text { Dépistage de base du VIH et des } \\
\text { ITS, et bilans hépatique et rénal. } \\
\text { Tests de suivi les deuxième } \\
\text { et quatrième semaines si des } \\
\text { symptômes se manifestent ou } \\
\text { si des anomalies de base sont } \\
\text { détectées. Dépistage du VIH 12 } \\
\text { semaines après l'exposition }\end{array}$ \\
\hline
\end{tabular}

Abréviation : ITS, infections transmises sexuellement

\section{Preuve pour la PrEP}

L'utilisation de TDF/FTC pour la PrEP chez les personnes séronégatives à haut risque continu d'infection au VIH a été rigoureusement évaluée dans des essais cliniques randomisés et s'est avérée être très efficace, en particulier chez les HARSAH (tableau 2). L'usage quotidien a été évalué dans deux essais cliniques, tandis que l'essai Ipergay étudiait la PrEP «sur demande », où le TDF/FTC était utilisé de deux à 24 heures avant l'exposition (dose de charge de deux comprimés) suivi d'un usage quotidien jusqu'à 48 heures après la dernière exposition sexuelle (19).

On a testé le TDF seul et le TDF/FTC pour la prévention de la transmission du VIH parmi les couples hétérosexuels sérodifférents et chez d'autres hétérosexuels dans les zones hautement endémiques (23-26). Les conclusions de ces études sont contradictoires. En effet, certaines études montrent des effets protecteurs et d'autres pas. Cette situation semble être principalement attribuable à une prise irrégulière des médicaments au cours de l'étude (26).

L'utilisation du TDF seul comme PrEP par les personnes s'injectant des drogues a démontré une diminution de 48,9\% de I'incidence du VIH dans l'ensemble, selon une étude réalisée à Bangkok (27). Une limitation de cette étude est que les aiguilles stériles n'ont pas pu être fournies aux participants à l'étude en vertu de la loi thaïlandaise, ce qui signifie que l'avantage supplémentaire de la PrEP demeure inconnu lorsque tout un ensemble de stratégies de prévention fondées sur des données probantes pour les personnes s'injectant des drogues est également mis en œuvre.
Tableau 2 : Résumé des essais de la prophylaxie préexposition contre le VIH chez les hommes qui ont des rapports sexuels avec des hommes (HARSAH)

\begin{tabular}{|c|c|c|c|c|c|}
\hline Étude & $\begin{array}{c}\text { Critères } \\
\text { d'inclusion }\end{array}$ & Intervention & $\begin{array}{c}\text { Taux } \\
\text { d'incidence } \\
\text { du VIH } \\
\text { (Groupe } \\
\text { témoin) }\end{array}$ & $\begin{array}{l}\text { Réduction } \\
\text { des risques }\end{array}$ & $\begin{array}{c}\text { Nombre } \\
\text { de sujets à } \\
\text { traiter par } \\
\text { année }\end{array}$ \\
\hline $\begin{array}{l}\text { iPrEx } \\
(20,21)\end{array}$ & $\begin{array}{l}\text { Risque élévé } \\
\text { pour le VIH } \\
\text { : Sexe anal } \\
\text { avec quatre } \\
\text { partenaires et } \\
\text { plus } \\
\text { RSANP avec } \\
\text { un partenaire } \\
\text { séropositif ou } \\
\text { incommun } \\
\text { Avant ITS } \\
\text { Rapports } \\
\text { sexuels } \\
\text { transactionnels }\end{array}$ & $\begin{array}{l}\text { Prise } \\
\text { quotidienne de } \\
\text { ténofovir } \\
\text { ou } \\
\text { d'emtricitabine } \\
\text { quotidien, ou } \\
\text { d'un placebo }\end{array}$ & $\begin{array}{l}3,9 / 100 \\
\text { années- } \\
\text { personnes } \\
\text { (IC à } 95 \% \text { : } \\
3,17 \text { à } 4,87 \text { ) }\end{array}$ & $\begin{array}{l}\text { Intention } \\
\text { de traiter : } \\
44 \% \\
\text { (IC } 95 \%: 15 \\
\text { à 63) } \\
\text { En } \\
\text { traitement } \\
\text { par un } \\
\text { médicament } \\
\text { détectable : } \\
92 \% \\
\text { (IC 95\% : } 40 \\
\text { à 99) }\end{array}$ & $\begin{array}{l}\text { Ayant eu } \\
\text { des } \\
\text { RSANP : } 36\end{array}$ \\
\hline $\begin{array}{l}\text { PROUD } \\
\text { (22) }\end{array}$ & $\begin{array}{l}\text { RSANP au } \\
\text { cours des } \\
\text { derniers } 90 \\
\text { jours }\end{array}$ & $\begin{array}{l}\text { Prise immédiate } \\
\text { ou quotidienne } \\
\text { différée de } \\
\text { ténofovir ou } \\
\text { d'emtricitabine }\end{array}$ & $\begin{array}{l}8.9 / 100 \mathrm{AP} \\
(\mathrm{IC} \text { à } 95 \% \\
: 6 \text { à } 12.7)\end{array}$ & $\begin{array}{l}\text { Total : } 86 \% \\
\text { (IC à } 95 \% \\
: 58 \text { à } 96)\end{array}$ & $\begin{array}{l}\text { Total :13 } \\
\text { (IC 95\% } \\
: 9 \text { à } 25)\end{array}$ \\
\hline $\begin{array}{l}\text { Ipergay } \\
\text { (19) }\end{array}$ & $\begin{array}{l}\text { RSNAP } \\
\text { avec deux } \\
\text { partenaires } \\
\text { et plus au } \\
\text { cours des six } \\
\text { derniers mois }\end{array}$ & $\begin{array}{l}\text { Ténofovir ou } \\
\text { emitricitabine } \\
\text { sur demand ou } \\
\text { placebo }\end{array}$ & $6,6 / 100 \mathrm{AP}$ & $\begin{array}{l}\text { Total: } 86 \% \\
\text { (IC } 95 \%: 40 \\
\text { à 99) }\end{array}$ & Overall: 18 \\
\hline
\end{tabular}

Abréviations: AP, années-personnes; IC, intervalle de confiance; ITS, infections transmises sexuellement; RSANP, relations sexuelles anales non protégées

\section{Défis et occasions pour la prévention biomédicale}

\section{Détermination des populations pour la PrEP}

La modélisation mathématique des études a clairement montré que la PrEP est plus rentable lorsqu'elle est administrée à des personnes à risque élevé au $\mathrm{VIH}(28,29)$. À ce jour, l'augmentation de la PrEP au Canada a surtout porté sur les HARSAH en raison de la disponibilité de données précises sur la façon de cerner les sous-populations à forte incidence et sur la faisabilité de mise en œuvre de la PrEP. L'utilisation de marqueurs cliniques, tels que les antécédents d'infections transmises sexuellement ou l'utilisation d'outils de calcul de risque validés (30-34) permet la détermination des HARSAH canadiens dont le taux d'incidence du VIH se situe bien au-delà des seuils recommandés au niveau international, soit de 2 à $3 \%$ par an $(35,36)$. La sensibilisation des HARSAH à la PrEP dans les zones urbaines a considérablement augmenté au cours des dernières années. Des études récentes ont montré que $91,3 \%$ des HARSAH ayant passé des tests anonymement dans une clinique de santé sexuelle à Toronto et que $80 \%$ des HARSAH participant à une étude de cohorte à Vancouver connaissaient l'existence de la PrEP (37).

Les projets de démonstration ont montré la possibilité d'obtenir une adhésion élevée et des données sur une mise en œuvre "dans le monde réel», ce qui laisse croire que la PrEP pourrait réduire considérablement l'épidémie du VIH. L'utilisation quotidienne du TDF/FTC a éliminé les nouvelles infections au $\mathrm{VIH}$ chez les HARSAH qui reçoivent la PrEP dans un organisme de soins de santé à San Francisco $(38,39)$. De même, I'utilisation de la PrEP comme une composante de services complets contre le $\mathrm{VIH}$ a mené à une réduction de $32 \%$ des nouveaux diagnostics 
dans des cliniques offrant des services aux HARSAH à Londres (6). En Nouvelle-Galles du Sud (Australie), une réduction de $40 \%$ des infections récentes au VIH chez les HARSAH a été attribuée à l'augmentation de l'utilisation de la PrEP (40).

L'utilisation chez les personnes s'injectant des drogues ou dans des situations de transmission hétérosexuelle présente également du potentiel, mais qui comporte des défis différents. Par exemple, il faudrait une intégration en temps réel avec des programmes de surveillance pour identifier les communautés où il y a des transmissions actives et de nouvelles stratégies pratiques seront nécessaires pour identifier ceux qui sont le plus à risque. L'intégration de la prévention biomédicale dans les stratégies de réduction des risques chez les personnes s'injectant des drogues, où l'impact évident sur la transmission du $\mathrm{VIH} \mathrm{a}$ déjà été démontré, n'a pas encore été évaluée. Une possibilité intéressante, qui est évaluée par le Partner Demonstration Project africain, consiste à offrir la PrEP aux partenaires séronégatifs des personnes nouvellement diagnostiquées qui commencent une TA. Elle sert de « raccourci » pendant que la suppression de charge virale est obtenue chez le partenaire séropositif (41). Cette stratégie peut être utile dans des communautés localisées dont la transmission du VIH est continue. Le Canada a besoin d'initiatives de mise en œuvre scientifiques similaires pour mieux comprendre l'acceptabilité, la faisabilité et les résultats sanitaires réels pendant que la PrEP est déployée dans les populations à risque élevé.

\section{Élargissement de l'accès à la PrEP}

Actuellement, la PrEP est prescrite principalement par des médecins spécialistes, mais cette pratique crée un goulot d'étranglement. L'élargissement de l'accès à la PrEP pourrait se faire au moyen de la création de capacités auprès des fournisseurs de soins primaires. Étant donné que les fournisseurs de soins primaires offrent déjà d'autres stratégies de prévention primaire des maladies chroniques basée sur des données probantes, la prescription de la PrEP est une extension logique de ce rôle.

Les sondages auprès de médecins soutiennent la notion que les soins primaires conviennent à la mise en œuvre de la $\operatorname{PrEP}(42)$. Les fournisseurs de soins primaires fournissent des soins longitudinaux à un grand nombre de personnes séronégatives à risque et possèdent des compétences dans d'autres composantes de la prévention du $\mathrm{VIH}$, comme l'orientation, la toxicomanie et la santé mentale.

Des études récentes effectuées aux États-Unis ont permis de constater que la sensibilisation chez les fournisseurs de soins primaires a augmenté dans les années suivant la publication de lignes directrices cliniques par les Centers for Disease Control and Prevention (de 76 à $93 \%$ sont au courant), de 17 à $34 \%$ des personnes interrogées prescrivant maintenant la $\operatorname{PrEP}(43,44,45)$. Contrairement aux États-Unis, à l'Australie et au Royaume-Uni, qui ont régulièrement mis à jour les lignes directrices nationales pour le traitement du VIH et la nPEP, le Canada n'a rien fait en ce sens. Cette situation résulte en partie du fait que les soins de santé sont de compétence provinciale ou territoriale. Actuellement, seuls la Colombie-Britannique et le Québec ont émis des directives cliniques pour les médecins prescrivant de la $\operatorname{PrEP}(46,47)$. En Colombie-Britannique, il n'y a pas de restrictions concernant le type de médecin qui peut prescrire la PrEP, tandis qu'au Québec, l'orientation actuelle suggère que la PrEP doit uniquement être prescrite par des médecins qui ont déjà fait l'expérience de la prescription de thérapie antirétrovirale.

Selon les médecins, le manque de connaissances générales sur la PrEP serait un obstacle potentiel à la prescription du médicament (44). D'autres obstacles pouvant empêcher I'adoption de la PrEP par les médecins pourraient comprendre les perceptions erronées au sujet de ses effets secondaires potentiels, les préoccupations concernant la compensation du risque (l'idée qu'on puisse adopter plus de comportements à risque en se croyant protégé contre le $\mathrm{VIH}$, ce qui éliminerait les avantages de la protection) et une augmentation des infections transmises sexuellement chez les utilisateurs de la PrEP. Ces obstacles peuvent être facilement traités (48) ou sont ouverts aux interventions de dépistage (49).

L'amélioration de l'éducation des médecins et les efforts déployés pour convaincre les médecins de soins primaires de prescrire ou de conseiller la PrEP contribueront à élargir la portée de cette stratégie de prévention primaire.

\section{Qu'en est-il des remboursements par l'État?}

Santé Canada a uniquement autorisé l'utilisation du TDF/ FTC, conjointement avec des pratiques sexuelles plus sûres, pour la prévention du VIH en 2016. Jusqu'à récemment, seul le Québec offrait le remboursement de la PrEP. Ailleurs, les personnes qui souhaitaient y avoir recours devaient se contenter de contracter une assurance privée de responsabilité civile ou de devenir membre d'un " club d'acheteurs " y donnant accès dans les pharmacies en ligne (50). En août 2016, dans le cadre du Programme commun d'évaluation des médicaments de l'Agence canadienne des médicaments et des technologies de la santé, le Comité consultatif canadien d'expertise sur les médicaments a recommandé officiellement aux régimes publics d'assurance-médicaments fédéral, provinciaux et territoriaux participants du Canada que le recours au TDF/FTC pour la PrEP soit ajouté sur leurs formulaires, à la suite de la recommandation de Santé Canada, si les deux conditions suivantes sont remplies :

- Que le TDF/FTC soit fourni dans le cadre d'un programme de santé sexuelle par un prescripteur ayant acquis de

l'expérience en traitement et en prévention de l'infection au $\mathrm{VIH} 1$

- Que le prix soit réduit (51)

Toutefois, la première condition est elle-même un obstacle à l'expansion de l'adoption de la PrEP, puisqu'elle implique que seuls les prescripteurs expérimentés en prévention ou en gestion du VIH devraient prescrire la PrEP.

Avec la très récente arrivée du TDF/FTC générique sur le marché canadien, des options pour réduire le prix des médicaments et en élargir l'accès sont déjà en place dans certaines régions. En septembre 2017, I'Ontario a annoncé la couverture de la PrEP par le Programme de médicaments de l'Ontario, sans aucune restriction sur le prescripteur. La Colombie-Britannique examine actuellement la couverture de la PrEP à la lumière des nouveaux prix des médicaments génériques (52). 


\section{Prochaines étapes}

Nous entrons dans une nouvelle ère dans les efforts d'élimination du VIH grâce à l'accès à des stratégies de prévention

biomédicale. Ces stratégies ont une efficacité prouvée chez les HARSAH, qui continuent d'être surreprésentés dans l'épidémie du VIH au Canada, et peuvent également avoir un rôle dans la diminution de la transmission chez d'autres populations à risque.

Pour tirer pleinement parti des avantages de la prévention biomédicale, un certain nombre de mesures sont nécessaires. La publication de lignes directrices cliniques permettrait de faire en sorte que les fournisseurs de soins de santé se sentent plus à I'aide de prescrire la PrEP. L'éducation médicale continue, ainsi que le résumé des données probantes accumulées sur la PrEP et l'accroissement de la sensibilisation aux lignes directrices, permettront d'améliorer les connaissances des prestataires de soins et d'ajouter peu à peu la PrEP au répertoire des services offerts par les fournisseurs de soins primaires. Dans les provinces où la PrEP est initialement fournie par ceux qui ont de l'expérience dans le traitement et dans la prévention de I'infection au VIH-1, d'autres cliniciens en soins primaires peuvent toujours identifier les personnes à risque, les renseigner sur la PrEP et offrir des possibilités d'orientation. La recommandation de la PrEP pourrait aussi être intégrée aux programmes sur les infections transmissibles sexuellement ou aux programmes de santé publique visant à avertir les partenaires, ou encore être associés aux programmes existants qui voient des personnes à risque se faire évaluer pour se prévaloir de la prophylaxie post-exposition. Des liens plus étroits entre les programmes de santé publique et les services cliniques pour la prévention et le traitement du VIH pourraient également améliorer la connaissance et l'acceptation de la PrEP.

\section{Conclusion}

Les stratégies de prévention biomédicale ont le potentiel de modifier la trajectoire de l'épidémie de VIH au Canada et dans le monde. L'intégration réussie de la PrEP dans les pratiques de prévention et de dépistage du VIH contribuera sans aucun doute à l'objectif global de l'élimination des nouvelles infections au $\mathrm{VIH}$.

\section{Déclaration des auteurs}

M.H. et D.T. ont conjointement conçu la rédaction de cet article et y ont contribué.

\section{Conflit d'intérêt}

M.H. : Il a reçu des honoraires pour la prestation de conférences éducatives qu'il avait lui-même conçues (Gilead, Merck et Janssen) ou pour la participation à des conseils consultatifs de BMS, Gilead, Merck, Janssen et Viiv Healthcare. Tous les montants ont été versés à son établissement.

D.T. : II a reçu des honoraires de Viiv Healthcare et de Merck pour la prestation de conférences éducatives qu'il avait lui-même conçues; son établissement a reçu de Gilead et Viiv Healthcare un soutien pour ses subventions de recherche; DT est enquêteur principal de site pour les essais cliniques parrainés par GSK.

\section{Références}

1. Centers for Disease Control (CDC). Kaposi's sarcoma and Pneumocystis pneumonia among homosexual men--New York City and California. MMWR Morb Mortal Wkly Rep 1981 Jul;30(25):305-8. PubMed (https://www.ncbi.nlm. nih.gov/entrez/query.fcgi?cmd=Retrieve\&db=PubMed\&li st_uids=6789108\&dopt=Abstract).

2. Centers for Disease Control (CDC). Pneumocystis pneumonia--Los Angeles. MMWR Morb Mortal Wkly Rep 1981 Jun;30(21):250-2. PubMed (https://www.ncbi.nlm. nih.gov/entrez/query.fcgi?cmd=Retrieve \&db=PubMed\&li st_uids=6265753\&dopt=Abstract).

3. Agence de la santé publique du Canada. Le VIH et le sida au Canada - Rapport de surveillance en date du 31 décembre 2014. Ottawa (ON): 2015. https://www.canada.ca/fr/santepublique/services/publications/maladies-et-affections/vihet-sida-canada-rapport-surveillance-31-decembre-2014.html [Consulté le 1 erfév 2017].

4. BC Centre for Excellence in HIV/AIDS. HIV monitoring quarterly report for Vancouver Coastal Health. Second Quarter 2016. http://www.cfenet.ubc.ca/sites/default/ files/uploads/publications/centredocs/vch_monitoring_

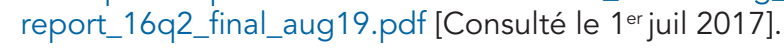

5. Hogg RS, Nosyk B, Harrigan PR, Lima VD, Chan K, Heath K et al. Rates of new infections in British Columbia continue to decline at a faster rate than in other Canadian regions. HIV Med 2013 Oct;14(9):581-2. DOI (http://dx.doi. org/10.1111/hiv.12079). PubMed (https://www.ncbi.nlm. nih.gov/entrez/query.fcgi?cmd=Retrieve\&db=PubMed\&li st_uids=24033869\&dopt=Abstract).

6. Brown AE, Mohammed H, Ogaz D, Kirwan PD, Yung M, Nash SG et al. Fall in new HIV diagnoses among men who have sex with men (MSM) at selected London sexual health clinics since early 2015: testing or treatment or pre-exposure prophylaxis (PrEP)? Euro Surveill 2017 Jun;22(25):30553. DOI (http://dx.doi.org/10.2807/15607917.ES.2017.22.25.30553). PubMed (https://www.ncbi.nlm. nih.gov/entrez/query.fcgi?cmd=Retrieve\&db=PubMed\&li st_uids=28662762\&dopt=Abstract).

7. Coates TJ, Richter L, Caceres C. Behavioural strategies to reduce HIV transmission: how to make them work better. Lancet 2008 Aug;372(9639):669-84. DOI (http://dx.doi. org/10.1016/S0140-6736(08)60886-7). PubMed (https://www. ncbi.nlm.nih.gov/entrez/query.fcgi?cmd=Retrieve \&db=PubM ed\&list_uids=18687459\&dopt=Abstract ).

8. Cohen MS, Chen YQ, McCauley M, Gamble T, Hosseinipour MC, Kumarasamy N et al.; HPTN 052 Study Team. Prevention of HIV-1 infection with early antiretroviral therapy. N Engl J Med 2011 Aug;365(6):493-505. DOl (http://dx.doi. org/10.1056/NEJMoa1105243). PubMed (https://www.ncbi. nlm.nih.gov/entrez/query.fcgi?cmd=Retrieve \&db=PubMed\&l ist_uids=21767103\&dopt=Abstract).

9. Rodger AJ, Cambiano V, Bruun T, Vernazza P, Collins S, van Lunzen J et al.; PARTNER Study Group. Sexual Activity Without Condoms and Risk of HIV Transmission in Serodifferent Couples When the HIV-Positive Partner Is Using Suppressive Antiretroviral Therapy. JAMA 2016 Jul;316(2):171-81. DOI (http://dx.doi.org/10.1001/ 
jama.2016.5148). PubMed (https://www.ncbi.nlm.nih. gov/entrez/query.fcgi?cmd=Retrieve\&db=PubMed\&lis t_uids=27404185\&dopt=Abstract)

10. Nosyk B, Zang X, Min JE, Krebs E, Lima VD, Milloy MJ et al. Relative effects of antiretroviral therapy and harm reduction initiatives on HIV incidence in British Columbia, Canada, 1996-2013: a modelling study. Lancet HIV 2017 Jul;4(7):e303-10. DOI (http://dx.doi.org/10.1016/S23523018(17)30045-0). PubMed (https://www.ncbi.nlm.nih. gov/entrez/query.fcgi?cmd=Retrieve \&db=PubMed\&lis t_uids=28366707\&dopt=Abstract).

11. Brown AE, Gill ON, Delpech VC. HIV treatment as prevention among men who have sex with men in the UK: is transmission controlled by universal access to HIV treatment and care? HIV Med 2013 Oct;14(9):563-70. DOI (http:// dx.doi.org/10.1111/hiv.12066). PubMed (https://www.ncbi. $\mathrm{nlm}$.nih.gov/entrez/query.fcgi?cmd=Retrieve \&db=PubMed\&l ist_uids=23890150\&dopt=Abstract).

12. Phillips AN, Cambiano V, Nakagawa F, Brown AE, Lampe $F$, Rodger A et al. Increased HIV incidence in men who have sex with men despite high levels of ART-induced viral suppression: analysis of an extensively documented epidemic. PLoS One 2013;8(2):e55312. DOl (https://doi. org/10.1371/journal.pone.0055312). PubMed (https://www. ncbi.nlm.nih.gov/entrez/query.fcgi?cmd=Retrieve\&db=PubM ed\&list_uids=23457467\&dopt=Abstract).

13. Chibo D, Kaye M, Birch C. HIV transmissions during seroconversion contribute significantly to new infections in men who have sex with men in Australia. AIDS Res Hum Retroviruses 2012 May;28(5):460-4. 10.1089/ AID.2011.0137. PubMed (https://www.ncbi.nlm.nih. gov/entrez/query.fcgi?cmd=Retrieve\&db=PubMed\&lis t_uids=21806486\&dopt=Abstract).

14. Brenner BG, Roger M, Stephens D, Moisi D, Hardy I, Weinberg J et al.; Montreal PHI Cohort Study Group. Transmission clustering drives the onward spread of the HIV epidemic among men who have sex with men in Quebec. J Infect Dis 2011 Oct;204(7):1115-9. DOI (http://dx.doi. org/10.1093/infdis/jir468). PubMed (https://www.ncbi.nlm. nih.gov/entrez/query.fcgi?cmd=Retrieve \&db=PubMed\&li st_uids=21881127\&dopt=Abstract).

15. Cáceres CF, Koechlin F, Goicochea P, Sow PS, O’Reilly $\mathrm{KR}$, Mayer $\mathrm{KH}$ et al. The promises and challenges of preexposure prophylaxis as part of the emerging paradigm of combination HIV prevention. J Int AIDS Soc 2015 Jul;18(4 Suppl 3):19949. 10.7448/IAS.18.4.19949. PubMed (https:// www.ncbi.nlm.nih.gov/entrez/query.fcgi?cmd=Retrieve\&db= PubMed\&list_uids=26198341\&dopt=Abstract).

16. Smith DK, Grohskopf LA, Black RJ, Auerbach JD, Veronese F, Struble KA et al.; U.S. Department of Health and Human Services. Antiretroviral postexposure prophylaxis after sexual, injection-drug use, or other nonoccupational exposure to HIV in the United States: recommendations from the U.S. Department of Health and Human Services. MMWR Recomm Rep 2005 Jan;54 RR-2:1-20. PubMed (https://www.ncbi.nlm. nih.gov/entrez/query.fcgi?cmd=Retrieve \&db=PubMed\&li st_uids=15660015\&dopt=Abstract).

17. Tsai CC, Emau P, Follis KE, Beck TW, Benveniste RE, Bischofberger $\mathrm{N}$ et al. Effectiveness of postinoculation
(R)-9-(2-phosphonylmethoxypropyl) adenine treatment for prevention of persistent simian immunodeficiency virus SIVmne infection depends critically on timing of initiation and duration of treatment. J Virol 1998 May;72(5):4265-73. PubMed (https://www.ncbi.nlm.nih. gov/entrez/query.fcgi?cmd=Retrieve\&db=PubMed\&lis t_uids=9557716\&dopt=Abstract).

18. Fonner VA, Dalglish SL, Kennedy CE, Baggaley R, O'Reilly $K R$, Koechlin FM et al. Effectiveness and safety of oral HIV preexposure prophylaxis for all populations. AIDS 2016 Jul;30(12):1973-83. DOI (http://dx.doi.org/10.1097/ QAD.0000000000001145). PubMed (https://www.ncbi.nlm. nih.gov/entrez/query.fcgi?cmd=Retrieve $\& d b=$ PubMed $\&$ li st_uids=27149090\&dopt=Abstract).

19. Molina JM, Capitant C, Spire B, Pialoux G, Cotte L, Charreau I et al.; ANRS IPERGAY Study Group. On-Demand Preexposure Prophylaxis in Men at High Risk for HIV-1 Infection. N Engl J Med 2015 Dec;373(23):2237-46. DOI (http://dx.doi.org/10.1056/NEJMoa1506273). PubMed (https://www.ncbi.nlm.nih.gov/entrez/query.fcgi?cmd=Retrie ve\&db=PubMed\&list_uids=27149090\&dopt=Abstract).

20. Grant RM, Lama JR, Anderson PL, McMahan V, Liu AY, Vargas $L$ et al.; iPrEx Study Team. Preexposure chemoprophylaxis for HIV prevention in men who have sex with men. $N$ Engl J Med 2010 Dec;363(27):2587-99. DOI (http://dx.doi. org/10.1056/NEJMoa1011205) PubMed (https://www.ncbi. $\mathrm{nlm}$.nih.gov/entrez/query.fcgi?cmd=Retrieve\&db=PubMed\&l ist_uids=21091279\&dopt=Abstract)

21. Buchbinder SP, Glidden DV, Liu AY, McMahan V, Guanira JV, Mayer $\mathrm{KH}$ et al. HIV pre-exposure prophylaxis in men who have sex with men and transgender women: a secondary analysis of a phase 3 randomised controlled efficacy trial. Lancet Infect Dis 2014 Jun;14(6):468-75. DOI (http://dx.doi. org/10.1016/S1473-3099(14)70025-8). PubMed (https://www. ncbi.nlm.nih.gov/entrez/query.fcgi?cmd=Retrieve \&db=PubM ed\&list_uids=24613084\&dopt=Abstract).

22. McCormack S, Dunn DT, Desai M, Dolling DI, Gafos M, Gilson R et al. Pre-exposure prophylaxis to prevent the acquisition of HIV-1 infection (PROUD): effectiveness results from the pilot phase of a pragmatic open-label randomised trial. Lancet 2016 Jan;387(10013):53-60. DOI (http://dx.doi. org/10.1016/S0140-6736(15)00056-2). PubMed (https://www. ncbi.nlm.nih.gov/entrez/query.fcgi?cmd=Retrieve\&db=PubM ed\&list_uids=26364263\&dopt=Abstract).

23. Baeten JM, Donnell D, Mugo NR, Ndase P, Thomas KK, Campbell JD et al.; Partners PrEP Study Team. Single-agent tenofovir versus combination emtricitabine plus tenofovir for pre-exposure prophylaxis for HIV-1 acquisition: an update of data from a randomised, double-blind, phase 3 trial. Lancet Infect Dis 2014 Nov;14(11):1055-64. DOI (http://dx.doi. org/10.1016/S1473-3099(14)70937-5) PubMed (https://www. ncbi.nlm.nih.gov/entrez/query.fcgi?cmd=Retrieve\&db=PubM ed\&list_uids=25300863\&dopt=Abstract).

24. Thigpen MC, Kebaabetswe PM, Paxton LA, Smith DK, Rose CE, Segolodi TM et al.; TDF2 Study Group. Antiretroviral preexposure prophylaxis for heterosexual HIV transmission in Botswana. N Engl J Med 2012 Aug;367(5):423-34. DOI (http://dx.doi.org/10.1056/ NEJMoa1110711). PubMed (https://www.ncbi.nlm.nih. 
gov/entrez/query.fcgi?cmd=Retrieve\& $\mathrm{db}=$ PubMed\&lis t_uids=22784038\&dopt=Abstract).

25. Van Damme L, Corneli A, Ahmed K, Agot K, Lombaard J, Kapiga S et al.; FEM-PrEP Study Group. Preexposure prophylaxis for HIV infection among African women. N Engl J Med 2012 Aug;367(5):411-22. DOl (http://dx.doi. org/10.1056/NEJMoa1202614). PubMed (https://www.ncbi. nlm.nih.gov/entrez/query.fcgi?cmd=Retrieve\&db=PubMed\&l ist_uids=22784040\&dopt=Abstract).

26. Marrazzo JM, Ramjee G, Richardson BA, Gomez K, Mgodi N, Nair G et al.; VOICE Study Team. Tenofovir-based preexposure prophylaxis for HIV infection among African women. N Engl J Med 2015 Feb;372(6):509-18. DOI (http:// dx.doi.org/10.1056/NEJMoa1402269) PubMed (https://www. ncbi.nlm.nih.gov/entrez/query.fcgi?cmd=Retrieve\&db=PubM ed\&list_uids=25651245\&dopt=Abstract).

27. Choopanya K, Martin M, Suntharasamai P, Sangkum U, Mock PA, Leethochawalit M et al.; Bangkok Tenofovir Study Group. Antiretroviral prophylaxis for HIV infection in injecting drug users in Bangkok, Thailand (the Bangkok Tenofovir Study): a randomised, double-blind, placebo-controlled phase 3 trial. Lancet 2013 Jun;381(9883):2083-90. DOI (http://dx.doi. org/10.1016/S0140-6736(13)61127-7). PubMed (https://www. ncbi.nlm.nih.gov/entrez/query.fcgi?cmd=Retrieve\&db=PubM ed\&list_uids=23769234\&dopt=Abstract).

28. Kugathasan H, Thavorn K, Moqueet N. Economic evaluations of HIV pre-exposure prophylaxis: A systematic review. 26th Annual Canadian Conference on HIV/AIDS Research Abstract EPH 106. Montreal, QC; 2017.

29. Gomez GB, Borquez A, Case KK, Wheelock A, Vassall $A$, Hankins $C$. The cost and impact of scaling up preexposure prophylaxis for HIV prevention: a systematic review of cost-effectiveness modelling studies. PLoS Med 2013;10(3):e1001401. DOI (https://doi.org/10.1371/ journal.pmed.1001401). PubMed (https://www.ncbi.nlm. nih.gov/entrez/query.fcgi?cmd=Retrieve \&db=PubMed\&li st_uids=23554579\&dopt=Abstract).

30. Pathela P, Braunstein SL, Blank S, Shepard C, Schillinger JA. The high risk of an HIV diagnosis following a diagnosis of syphilis: a population-level analysis of New York City men. Clin Infect Dis 2015 Jul;61(2):281-7. DOI (http://dx.doi. org/10.1093/cid/civ289). PubMed (https://www.ncbi.nlm. nih.gov/entrez/query.fcgi?cmd=Retrieve $\& d b=$ PubMed $\&$ li st_uids=25870333\&dopt=Abstract).

31. Smith DK, Pals SL, Herbst JH, Shinde S, Carey JW. Development of a clinical screening index predictive of incident HIV infection among men who have sex with men in the United States. J Acquir Immune Defic Syndr 2012 Aug;60(4):421-7. DOl (http://dx.doi.org/10.1097/ QAl.0b013e318256b2f6). PubMed (https://www.ncbi.nlm. nih.gov/entrez/query.fcgi?cmd=Retrieve\&db=PubMed\&li st_uids=22487585\&dopt=Abstract).

32. Lachowsky N, Zui C, Serada P. HIV Incidence Rate and Predictors Among Gay and other Men who have Sex with Men (MSM) in Vancouver: Additional Benefit of an Administrative Health Data Linkage. 25th Annual Canadian Conference on HIV/AIDS Research. Winnipeg 12 -15 May 2016. Abstract EPHP5.04.
33. Samji H, Hu J, Moore D. HIV Incidence among Gay, Bisexual, and other Men who Have Sex with Men Attending Sexually Transmitted Infection Clinics in British Columbia. 25th Annual Canadian Conference on HIV/AIDS Research. Winnipeg May 12- 15 2015. Abstract EPHP 2.02.

34. Wilton J, Kain T, Fowler S, Hart TA, Grennan T, Maxwell J et al. Use of an HIV-risk screening tool to identify optimal candidates for PrEP scale-up among men who have sex with men in Toronto, Canada: disconnect between objective and subjective HIV risk. J Int AIDS Soc 2016 Jun;19(1):20777. DOI (http://dx.doi.org/10.7448/IAS.19.1.20777). PubMed (https://www.ncbi.nlm.nih.gov/entrez/query.fcgi?cmd=Retrie ve\&db=PubMed\&list_uids=27265490\&dopt=Abstract).

35. World Health Organization. Guideline on when to start antiretroviral therapy and on pre-exposure prophylaxis for HIV. September 2015. http://www.who.int/hiv/pub/ guidelines/earlyrelease-arv/en/ [Consulté le 22 juil 2016].

36. Günthard HF, Saag MS, Benson CA, del Rio C, Eron JJ, Gallant JE et al. Antiretroviral Drugs for Treatment and Prevention of HIV Infection in Adults: 2016 Recommendations of the International Antiviral Society-USA Panel. JAMA 2016 Jul;316(2):191-210. DOI (http://dx.doi. org/10.1001/jama.2016.8900). PubMed (https://www.ncbi. $\mathrm{nlm}$.nih.gov/entrez/query.fcgi?cmd=Retrieve \&db=PubMed\&| ist_uids=27404187\&dopt=Abstract).

37. Khaketla M, Lachowsky NH. Four year trends in awareness and use of PrEP in gbMSM Vancouver, Canada. CROI 2017. Abstract 966.

38. Tan DH, Shubb A, Lawless J. High adherence but modest risk compensation in a PrEP demonstration project. 25th Annual Canadian Conference on HIV/AIDS Research Abstract MD 11. Winnipeg, Canada. 2016.

39. Volk JE, Marcus JL, Phengrasamy T, Blechinger D, Nguyen DP, Follansbee $S$ et al. No New HIV Infections With Increasing Use of HIV Preexposure Prophylaxis in a Clinical Practice Setting. Clin Infect Dis 2015 Nov;61(10):1601-3. DOI (http://dx.doi.org/10.1093/cid/civ778). PubMed (https:// www.ncbi.nlm.nih.gov/entrez/query.fcgi?cmd=Retrieve\&db= PubMed\&list_uids=26334052\&dopt=Abstract).

40. Cairns G. New South Wales reports record drop in HIV diagnoses: fewest this year since records began. AIDSMAP 25 Aug 2017. http://www.aidsmap.com/New-South-Walesreports-record-drop-in-HIV-diagnoses-fewest-this-year-sincerecords-began/page/3167508 [Accessed October 8, 2017].

41. Baeten JM, Heffron R, Kidoguchi L, Mugo NR, Katabira E, Bukusi EA et al.; Partners Demonstration Project Team. Integrated Delivery of Antiretroviral Treatment and Preexposure Prophylaxis to HIV-1-Serodiscordant Couples: A Prospective Implementation Study in Kenya and Uganda. PLoS Med 2016 Aug;13(8):e1002099. DOI (http://dx.doi. org/10.1371/journal.pmed.1002099). PubMed (h.ttps://www. ncbi.nlm.nih.gov/entrez/query.fcgi?cmd=Retrieve\&db=PubM ed\&list_uids=27552090\&dopt=Abstract)

42. Hoffman S, Guidry JA, Collier KL, Mantell JE, BoccherLattimore D, Kaighobadi F et al. A Clinical Home for Preexposure Prophylaxis: Diverse Health Care Providers' Perspectives on the "Purview Paradox". J Int Assoc Provid AIDS Care 2016 Jan-Feb;15(1):59-65. DOI (http://dx.doi. 
org/10.1177/2325957415600798). PubMed (https://www. ncbi.nlm.nih.gov/entrez/query.fcgi?cmd=Retrieve\&db=PubM ed\&list_uids=26293904\&dopt=Abstract).

43. US Public Health Service. Pre-exposure prophylaxis for the prevention of HIV infection in the United States - 2014. A clinical practice guideline. https://www.cdc.gov/hiv/pdf/

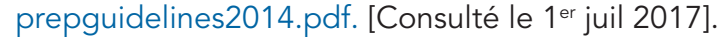

44. Petroll AE, Walsh JL, Owczarzak JL, McAuliffe TL, Bogart LM, Kelly JA. PrEP Awareness, Familiarity, Comfort, and Prescribing Experience among US Primary Care Providers and HIV Specialists. AIDS Behav 2017 May;21(5):1256-67. DOI (http://dx.doi.org/10.1007/ s10461-016-1625-1). PubMed (https://www.ncbi.nlm.nih. gov/entrez/query.fcgi?cmd=Retrieve\&db=PubMed\&lis t_uids=27885552\&dopt=Abstract).

45. Blackstock OJ, Moore BA, Berkenblit GV, Calabrese SK, Cunningham CO, Fiellin DA et al. A Cross-Sectional Online Survey of HIV Pre-Exposure Prophylaxis Adoption Among Primary Care Physicians. J Gen Intern Med 2017 Jan;32(1):62-70. DOI (http://dx.doi.org/10.1007/ s11606-016-3903-z). PubMed (https://www.ncbi.nlm.nih. gov/entrez/query.fcgi?cmd=Retrieve\&db=PubMed\&lis t_uids=27778215\&dopt=Abstract).

46. BC Centre for Excellence in HIV/AIDS. Guidance for the use of Pre-Exposure Prophylaxis (PrEP) for the prevention of HIV acquisition in British Columbia. Vancouver (BC); October 11, 2016. http://www.cfenet.ubc.ca/publications/centredocuments/guidance-use-pre-exposure-prophylaxis-prepprevention-hiv-acquisition. [Consulté le $1^{\text {er }}$ juil 2017].

47. Ministere de la Sante et des Services sociaux. Avis interimaire sur la prophylaxie preexposition au virus de I'immunodeficience humaine. 2013. publications.msss.gouv.
qc.ca/acrobat/f/documentation/2013/13-308-03W.pdf.

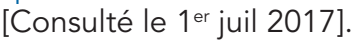

48. Yacoub R, Nadkarni GN, Weikum D, Konstantinidis I, Boueilh A, Grant RM et al. Elevations in Serum Creatinine With Tenofovir-Based HIV Pre-Exposure Prophylaxis: A Meta-Analysis of Randomized PlaceboControlled Trials. J Acquir Immune Defic Syndr 2016 Apr;71(4):e115-8. DOI (http://dx.doi.org/10.1097/ QAl.0000000000000906). PubMed (https://www.ncbi.nlm. nih.gov/entrez/query.fcgi?cmd=Retrieve $\& d b=$ PubMed\&li st_uids=26627105\&dopt=Abstract).

49. Cohen S, Vittinghoff E, Philip SS, Doblecki-Lewis S, Bacon O, Chege W et al. Quarterly STI Screening Optimizes STI Detection Among PrEP Users in the Demo Project. In Abstracts and Proceedings of the Conference on Retroviruses and Opportunistic Infections. Boston, MA. February 22-25, 2016. Abstract 870.

50. Davie Buyers Club. How to access PrEP without insurance in Vancouver (for \$45 CAD a month). https://daviebuyersclub. wordpress.com/. [Consulté le 31 août juil 2017].

51. Canadian Agency for Drugs and Technologies in Health. CADTH Canadian Drug Expert Committee Final Recommendation: Emtricitbaine/Tenofovir disoproxil fumarate. Common Drug Review; Notice of Final Recommendation-Aug 24, 2016. https://www.cadth.ca/ sites/default/files/cdr/complete/SR0479_complete_Truvada_ Aug-26-16.pdf

52. Ontario to cover HIV prevention pill under public health plan. CBC News Posted Sep22, 2017. http://www.cbc.ca/ news/health/hiv-prep-coverage-1.4302184. health/hiv-prepcoverage-1.4302184. [Consulté le 8 oct 2017]. 